Forum Presentation

\title{
Plaque Morphology of Acute Coronary Syndrome
}

\author{
Ryohei Hisaki and Chikao Yutani
}

Department of Pathology, National Cardiovascular Center, Osaka, Japan.

\begin{abstract}
Background : There have been many histopathological studies of acute coronary syndromes (ACS). As for the relation between plaque morphology of culprit lesion and clinical background in ACS, few data are available. Purpose: We compared the incidence, morphological characteristics and clinical background associated with plaque rupture versus thrombosis in eroded plaques without rupture. Materials and Methods : We defined ACS as sudden coronary death, acute myocardial infarction, and unstable angina. One hundred twenty-two consecutive cases with intracoronary thrombi dying within one month after onset were histologically studied. After fixation at $100 \mathrm{mmHg}$, the major epicardial coronary arteries were cut transversely. All segments were studied by histological examination and morphological measurements. Results : Plaque rupture was identified in $\mathbf{7 0}$ lesions and erosion were in $\mathbf{5 4}$ lesions. The plaque rupture group had larger lipid core and smaller calcium deposits than the erosion group. Hypertensives had larger lipid core than normotensives. And diabetics had larger calcium deposits than non-diabetics. Also, smokers had larger lipid core and smaller calcium deposits than non-smokers. Conclusion : Coronary risk factors, i.e., hypertension, diabetes mellitus and smoking are associated with plaque morphology. Also, the area of the lipid core and calcium deposits of coronary arteries associated with pathogenesis of plaque thrombosis. $J$ Atheroscler Thromb, $1998 ; 4$ : $156-161$
\end{abstract}

Key words : Plaque disruption, Plaque rupture, Plaque erosion, Risk factor,

\section{Introduction}

Atherosclerotic coronary arteries consist of the intraarterial accumulation of variable quantities of intracellular and extracellular lipid, macrophage, $\mathrm{T}$ cells, smooth muscle cells, proteoglycan, collagen, calcium, and necrotic debris. Sudden changes in coronary plaque luminal surface morphology consisting of plaque rupture or erosion have been recognized as an important mechanism of thrombosis and in the clinical presentation of acute coronary syndromes (1-4).

A typical vulnerable plaque has been reported to consist of an eccentric plaque that is rich in extracellular lipid

Address for correspondence: Ryohei Hisaki, MD, Department of Pathology, National Cardiovascular Center, 5-7-1, Fujishirodai, Suita, Osaka 565-8565, Japan.

This article is presented in the Forum \#16 on Plaque Lesions and the Stabilization Factors at the 29th Annual Meeting on the Japan Atherosclerosis Society in Tokyo, 1997.

Received March 20, 1998. within a large lipid pool with a thin overlying fibrous cap (5-8). In these lesions, the fibrous cap is frequently infiltrated with activated macrophages and $T$ cells. Plaque disruption of a thin fibrous cap resulting in exposure of blood to the lipid core is believed to consist of the vast majority of coronary thrombi. This conclusion has emerged from studies of diverse populations that included indivisuals with sudden and nonsudden death with thrombi of main young ages (9-12). The morphology of the underlying plaque associated with the thrombus has been examined in varying degrees of detail. The purpose of this study was to describe the morphological feature and clinical background of coronary thrombosis in Japanese elder persons.

\section{Methods}

\section{Selection of Cases}

The necropsy records of the department of Pathology, National Cardiovascular Center were searched for 
patients coded as ACS including sudden death and acute myocardial infarction. We selected the cases who died within 4 weeks after onset of acute myocardial infarction, and in whom there are histologically intraluminal thrombi and acute myocardial infarct in distributional area of left ventricular wall. Cases who were performed percutaneous transvenous coronary angioplasty or coronary arterial bypass graft and were diagnosed with Kawasaki disease or intracoronary thromboemboli were excluded.

\section{Coronary Artery Evaluation}

All hearts were examined fresh and uncut, with fixation performed on the time of autopsy. The coronary arterial tree was perfusion-fixed with $10 \%$ neutral-buffered formalin overnight at $100 \mathrm{mmHg}$. Postmortem angiography with barium gelatin was performed in cases of sudden death who were suspected with acute coronary disease. The major epicardial coronary arteries (left main, left anterior descending, left circumflex, and right coronary arteries) and their major branches (left diagonals, left obtuse marginals, and posterior descending coronary artery) were cut transversely at approximately $3-\mathrm{mm}$ intervals (following decalcification if necessary). All segments were submitted for light microscopy, histological examination, and morphological measurements. Arterial segments were dehydrated in a series of graded alcohols, cleared with xylene, embedded in paraffin, cut at $4 \mathrm{~mm}$, and stained with hematoxylin-eosin.

For culprit lesion, additional staining with Masson trichrome and elastic van Gieson were performed. With Masson trichrome, collagen fibers and proteoglycan stain blue, and with elastic van Gieson, elastic fiber stains black, collagen fibers stain red.

Coronary thrombosis cases were separated into two categories based on the morphology of the underlying plaque following as Farb's classification (4). (1) Coronary thrombosis with plaque rupture was defined as a disruption of a fibrous cap over a lipid core with contact of the acute thrombi with the lipid pool. (2) Coronary thrombosis with superficial erosion (without plaque rupture) consisted of an acute thrombi in direct contact with the intimal plaque without rupture of a lipid pool. Segments that demonstrated luminal thrombi but no rupture into a lipid core underwent serial step-sectioning and staining (at every $40 \mathrm{~mm}$ for at least 1 to $2 \mathrm{~cm}$ ) to determine whether rupture of a fibrous cap was present deeper in the section.

The most severely narrowed arterial segment containing the thrombi was magnified and digitized. From this segment, the arterial size (defined by the area within the internal elastic lamina), lipid core area, and calcium deposits area were measured $(\mathrm{NIH}$ image software, version 1.56).

\section{Myocardial Evaluation}

The right and left ventricles were cut at $1.0-\mathrm{cm}$ intervals parallel to the posterior AV groove from apex to the base, and stained with hematoxylin-eosin and Masson trichrome stain. The myocardium was examined histologically for the presence of healed and/or acute myocardial infarction, at least one section of myocardium (from the large ventricular slice) from both left and right ventricles. Acute myocardial infarction was diagnosed by the presence of coagulation necrosis of myocytes for within 3 days, granulation tissue with inflammatory infiltrate for within 1 weeks, loose collagen fiber with inflammatory infiltrate for within 4 weeks. For evaluation of myocardium, clinical data was available.

\section{Risk Factor Evaluation}

Risk factors of each cases were analyzed for hypertension, diabetes mellitus and smoking. We defined hypertensives who abused antihypertensive drug or had past history for over $140 \mathrm{mmHg}$ at systolic blood pressure or 95 $\mathrm{mmHg}$ in outcome at least twice, and cases with diabetes mellitus who had anti-diabetic therapy including drug abuse, exercise, and diet, smoker whom we could take information from patient or family, and with hypercholesterolemia who had history that serum total cholesterol had been $220 \mathrm{mg} / \mathrm{dL}$ or higher.

\section{Statistical Analysis}

Numerical data are presented as mean \pm SD. Continuous variables were compared with Welch's $t$-test, and categorical variables were compared with a chi's squared test. A value of $\mathrm{P}<0.05$ was considered statistically significant.

\section{Results}

One hundred twenty-four lesions of 122 cases had an acute thrombi in a major epicardial coronary arteries. The population consisted of 95 men and 29 women (mean age, men $67.7 \pm 11.2$ and women $72.3 \pm 8.1, p<0.05$ ). As for clinical background, smoking men were 76 per 88 , women 6 per $28(p<0.001)$, also diabetic men were 35 per 88 , women 17 per $27(p<0.05)$. (Table 1)

\section{Coronary Pathology}

Of the 124 acutely thrombosed coronary artery plaques, there were 54 superficial erosions and 70 ruptures. In plaque rupture cases thin collagenous fibrous cap separated the lumen from the underlying lipid core (Fig. 1). In coronary thrombosis without rupture into lipid core, the plaque luminal surface was irregular, eroded, and lacked endothelial cells (Fig. 2).

In applying the definitions of advanced atherosclerotic lesions by the AHA Council on Arteriosclerosis (13-15), all lesions are type $\mathrm{VI}$ on the basis of the presence of an acute thrombi. However, the underlying plaque of cases with fibrous rupture would fit a type IV in ten, a type $\mathrm{Va}$ in 59 , a type $\mathrm{Vb}$ in one. The underlying plaque in the 
Table 1. Profile of subjects

\begin{tabular}{ccccccc}
\hline & Age (y.o.) & & HT & Smoking & DM & HC \\
\hline Male & & $(+)$ & 70 & 76 & 35 & 40 \\
$(\mathrm{n}=95)$ & $67.7 \pm 11.1$ & $(-)$ & 18 & 15 & 53 & 46 \\
& & unknown & 7 & 4 & 7 & 9 \\
Female & & $(+)$ & 19 & 6 & 17 & 11 \\
(n=29) & $72.3 \pm 8.1$ & $(-)$ & 8 & 22 & 10 & 15 \\
& & unknown & 2 & 2 & 3 \\
p-value & $<0.05$ & & N.S. & $<0.001$ & $<0.05$ & N.S. \\
\hline
\end{tabular}

HT : Hypertension, DM : Dibetes mellitus, HC : Hypercholesterolemia

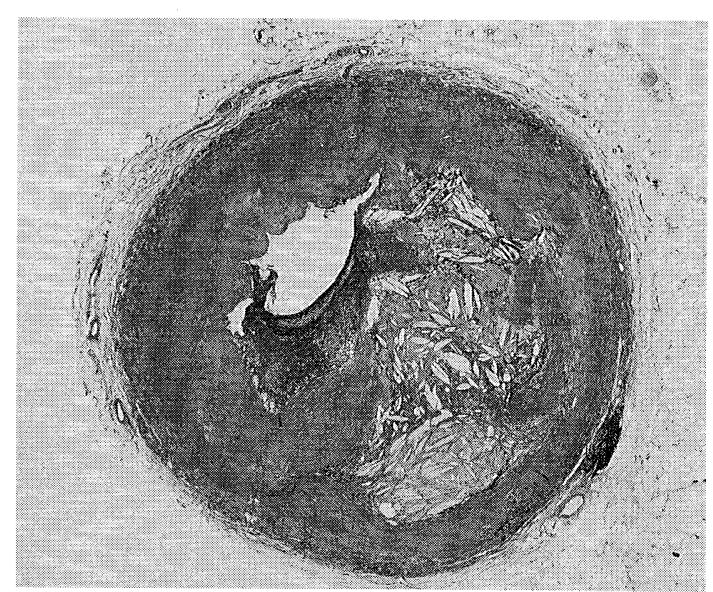

Fig. 1. Plaque rupture into lipid core (Masson trichrome; $\times 20$ ).

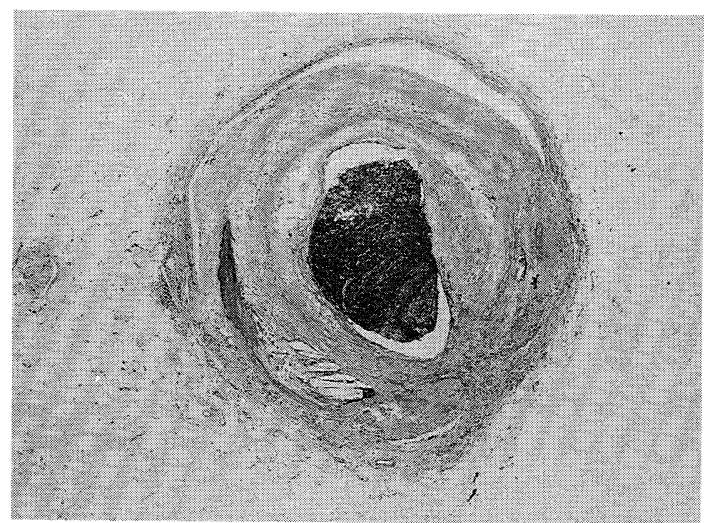

Fig. 2. Plaque erosion without rupture (Hematoxylineosin; $\times 20$ ).

superficially eroded arteries is a type III in three cases, a type IV in one, a type $\mathrm{Va}$ in 35 , a type $\mathrm{Vb}$ in 12 , a type $\mathrm{Vc}$ in three. (Table 2)

\section{Morphological Analysis of Plaque Disruption}

The mean percent luminal area stenosis in cases of coronary thrombosis due to plaque rupture was $85.0 \pm$
Table 2. Underlying plaque of coronary thrombosis - compared plaque erosion with plaque rupture into lipid core-

\begin{tabular}{lccc}
\hline Stary & $\begin{array}{c}\text { Plaque } \\
\text { erosion }\end{array}$ & $\begin{array}{c}\text { Plaque rupture } \\
\text { into lipid core }\end{array}$ & Total \\
\hline $\mathrm{VI} / \mathrm{III}$ & 3 & 0 & 3 \\
$\mathrm{VI} / \mathrm{IV}$ & 1 & 10 & 11 \\
$\mathrm{VI} / \mathrm{Va}$ & 35 & 59 & 94 \\
$\mathrm{VI} / \mathrm{Vb}$ & 12 & 1 & 13 \\
$\mathrm{VI} / \mathrm{Vc}$ & 3 & 0 & 3 \\
Total & 54 & 70 & 124 \\
\hline
\end{tabular}

$9.2 \%$ versus $85.2 \pm 12.3 \%$ in erosion. In the plaque rupture group, mean percent cross-sectional lipid core area was $42.5 \pm 16.4 \%$ versus $31.0 \pm 19.3 \%$ in eroded plaque $(p<0.001)$. The mean percent cross-sectional area calcium deposits was greater in greater in erodedplaque group $8.6 \pm 13.8 \%$ versus $2.8 \pm 5.2 \%$ in plaquerupture group $(p<0.01)$. (Table 3 )

\section{Clinical Background of Plaque Disruption}

Table 2 shows clinical background of 124 arteries and morphological analysis.

Hypertensives had larger lipid core $41.0 \pm 17.1 \%$ versus $27.1 \pm 19.9 \%$ in normotensives $(p<0.005)$. And diabetics had larger calcium deposits $8.3 \pm 13.0 \%$ than non-diabetics $3.7 \pm 8.1 \% \quad(p<0.05)$. Also smokers had larger lipid core $40.3 \pm 19.4 \%$ and smaller calcium deposits $3.6 \pm 7.6 \%$ versus non-smokers $31.1 \pm 15.3 \%, 10.3 \pm 14.6 \%$, respectively (lipid core; $p<0.01$, calcium deposits; $p<0.05$ ). The effect of past history of hypercholesterolemia on plaque morphology did not found in this history. (Table 4)

\section{Discussion}

The relationship between clinical background such as hypertension, hyperlipidemia, smoking and diabetes mellitus and ischemic coronary heart disease has been already reported in numerous articles (16-19). In these articles, however, few reports described how such as clinical backgrounds influence histopathologically on the mechanism of ACS. In present study, we obtained the following results, 1) Hypertensives have larger lipid core 
Table 3. Plaque morphology and pattern of disruption

\begin{tabular}{cccc}
\hline & Stenosis (\%) & Lipid core (\%) & Calcium deposits (\%) \\
\hline Plaque erosion & $85.2 \pm 12.3$ & $31.0 \pm 19.3$ & $8.6 \pm 13.8$ \\
Plaque rupture & $85.0 \pm 9.2$ & $42.5 \pm 16.4$ & $2.8 \pm 5.2$ \\
into lipid core & N.S. & $<0.001$ & $<0.01$ \\
P-value & & & \\
\hline
\end{tabular}

Table 4. Effects of risk factors to plaque morphology

\begin{tabular}{lllc}
\hline & Stenosis $(\%)$ & Lipid Core(\%) & $\begin{array}{c}\text { Calcium } \\
\text { Deposits }(\%)\end{array}$ \\
\hline$H T(+)$ & $85.4 \pm 11.0$ & $41.0 \pm 17.1^{\# 1}$ & $5.0 \pm 10.7$ \\
HT (-) & $84.5 \pm 9.4$ & $27.1 \pm 19.9^{\# 2}$ & $6.9 \pm 9.8$ \\
DM (+) & $87.0 \pm 9.7$ & $35.9 \pm 19.4$ & $8.3 \pm 13.0^{\# 5}$ \\
DM (-) & $83.0 \pm 11.3$ & $39.4 \pm 17.5$ & $3.7 \pm 8.1^{\# 6}$ \\
Smoker & $84.6 \pm 9.7$ & $40.3 \pm 19.4^{\# 3}$ & $3.6 \pm 7.6^{\# 7}$ \\
Non-smoker & $84.9 \pm 12.5$ & $31.1 \pm 15.3^{\sharp 4}$ & $10.3 \pm 14.6^{\sharp 8}$ \\
HC (+) & $87.0 \pm 10.0$ & $41.1 \pm 17.9$ & $4.3 \pm 9.1$ \\
HC (-) & $82.7 \pm 11.1$ & $35.0 \pm 19.4$ & $7.1 \pm 12.2$ \\
\hline
\end{tabular}

HT : Hypertension, DM : Diabetes mellitus, HC : hypercholesterolemia

$\# 1$ vs. \#2: $\mathrm{P}<0.005 \quad \# 3$ vs. \#4: $\mathrm{P}<0.01$

$\# 5$ vs. \#6: $\mathrm{P}<0.05 \quad \# 7$ vs. \#8: $\mathrm{P}<0.05$

than normotensives. 2) Diabetic patients have more calcium deposits than non-diabetic patients. 3) Smokers have have larger lipid core but less calcium deposits than non-smokers.

First of all, as to the positive relation of history of hypertension to the area of lipid core, endothelial injuries due to hypertension may increase their permeability leading to acceleration of LDL intake and monocyte adhesion $(20,21)$. Second, significant relation between history of diabetes mellitus and area of calcification may support that hyperinsulinemia, disturbance of lipid metabolism, and obesity associated with diabetes mellitus may cause systemic atherosclerosis including calcification. We already reported that calcification of coronary atherosclerosis in patients with diabetes mellitus was severe and diffuse until peripheral branches more significantly rather than non-diabetics (22).

Third, this study demonstrates that cigarette smoking is significantly associated with area of lipid core and negatively correlate to the area percent of calcification. Cigarette smoking was associated with decrease of $\mathrm{HDL}$ and influence of coronary blood flow, which may cause endothelial dysfunction and accerate lipid intake into the wall, subsequently enlargement of lipid core area $(23,24)$. Numerous reports $(18,25,26)$ mentioned that cigarette smoking was an important risk factor for coronary heart disease. Cigarette smoking also related to thrombosis regardless of the mechanism of plaque disruption(erosion or rupture). According to Burke et al. (18), the mechanism of coronary thrombosis in cigarette smokers may be related to increased platelet aggregation and plasma epinephrine concentration.

Although hypercholesterolemia is well known to be major risk factor for coronary atherosclerosis (18, 27-29), our study demonstrates that hypercholesterolemia does not correlate to the morphology of plaque disruption in ACS. Wagner et al. (30). mentioned that the lipid composition of the regressed plaque by cholesterol lowering treatment, suggested to change in plaque lipid into the character of extracellular deposits regarded as crystalline in rhesus monkeys.

As serum cholesterol normalizing, characteristics of plaque would be stabilized converting ester-cholesterol to cholesterol crystal, however, decreasing of plaque bulk is not established (31). Accordingly, serum value of cholesterol is progressive factor of atherosclerosis but does not effect neither stenosis nor the area of lipid core. Also, Burke et al. (18) mentioned that hyperlipidemia is not a direct risk factor of plaque disruption, but indirect factor via stimulating of macrophages. This fact answers why plaque rupture tend occurs in plaque with less severe stenosis. So cholesterol lowering therapy is one of the most effective strategies to prevent plaque rupture by stabilizing macrophages.

Additionally, shear stress, arterial stenosis and hemodynamic factor have been suspected to be triggers of plaque disruption (8).

By the way, as to pattern of disruption, present study demonstrates ruptured plaques trend to have larger lipid core, and smaller calcium deposits, significantly. We suspect that calcium deposits are characteristics of atherosclerosis but large calcium deposits defend to plaque rupture by replacing lipid core.

We conclude that the history of hypertension and smoking effect positively to the size of lipid core and that of diabetes mellitus to calcium deposits. Also, athero- 
sclerotic plaques with larger lipid core or smaller calcium deposits tend to occur plaque rupture into lipid core.

\section{References}

(1) Davies MJ and Thomas AC: Plaque fissuring: The cause of acute myocardial infarction, sudden ischaemic death, and crescendo angina. Br Heart J, 53 : 363-373, 1985

(2) Falk E : Coronary thrombosis : pathogenesis and clinical manifestations. Am J Cardiol, 68: 28B-35B, 1991

(3) Fuster V, Badimon L, Badimon JJ, and Chesebro JH: The pathogenesis of coronary artery disease and the acute coronary syndromes. N Engl J Med, 326: 242250, 310-318, 1992

(4) Farb A, Burke AP, Tang AL, Liang Y, Mannan P, Smialek $\mathrm{J}$, and Virmani $\mathrm{R}$ : Coronary plaque erosion without rupture into a lipid core. A frequent cause of coronary thrombosis in sudden coronary death. Circulation, 93 : 1354-1363, 1996

(5) Falk E : Morphologic feature of unstable atherothrombotic plaque underlying acute coronary syndromes. $\mathrm{Am} \mathrm{J}$ Cardiol, 63: 114E-120E, 1989

(6) Davies MJ: A macro and micro view of coronary vascular insult in ischemic heart disease. Circulation, 82(Suppl II) : II-38-II-46, 1990

(7) Fuster V, Stein B, Ambrose JA, Badimon L, Badimon JJ, and Chesebro $\mathrm{JH}$ : Atherosclerotic plaque rupture and thrombosis: evolving concepts. Circulation, 82(Suppl II) : II-47-II-59, 1990

(8) Falk E, Shah PK, and Fuster V : Coronary plaque disruption. Circulation, 92: 657-671, 1995

(9) Horie T, Sekiguchi M, and Hirosawa K : Coronary thrombosis in pathogenesis of myocardial infarction: histological study of coronary arteries in 108 necropsied cases using serial section. $\mathrm{Br}$ Heart $\mathrm{J}, 40$ : 153-161, 1978

(10) Falk E : Plaque rupture with severe pre-existing stenosis precipitating coronary thrombosis: characteristics of coronary atherosclerotic plaques underlying fatal occlusive thrombi. Br Heart J, $50: 127-134,1983$

(11) Davies MJ and Thomas A : Thrombosis and acute coronary-artery lesions in sudden cardiac ischemic death. N Engl J Med, 310: 1137-1140, 1984

(12) Davies MJ, Bland JM, Hangartner JRW, Angelini A, and Thomas AC: Factors influencing the presence or absence of acute coronary artery thrombi in sudden ischaemic death. Eur Heart J, 10: 203-208, 1989

(13) Stary $\mathrm{HC}$, Blankenhorn $\mathrm{DH}$, Chandler $\mathrm{AB}$, and et al: $\mathrm{A}$ definition of the intima of human arteries and of its atherosclerosis-prone regions : a report from the Committee on Vascular Lesions of the Council on Arteriosclerosis, American Heart Association. Circulation, 85 : 391-405, 1992

(14) Stary HC, Chandler AB, Glagov S, and et al: A definition of initial, fatty streak, and intermediate lesions of atherosclerosis: a report from the Committee on Vascular Lesions of the Council on Arteriosclerosis, American Heart Association. Circulation, 89: 2462-2478, 1994

(15) Stary $\mathrm{HC}$, Chandler AB, Dinsmore RE, and et al: A definition of advanced lesions and a histological classification of atherosclerosis : a report from the Committee on Vascular Lesions of the Council on Arteriosclerosis,
American Heart Association. Circulation, 92: 13551374, 1995.

(16) Clarkson TB, Prichard RW, Morgan TM, Petrick GS, and Klein KP : Remodeling of coronary arteries in human and nonhuman primates. JAMA, 271: 289-294, 1994

(17) PDAY Research Group : Relationship of atherosclerosis in young men to serum lipoprotein cholesterol concentration and smoking. JAMA, 264 : 3018-3024, 1990

(18) Burke AP, Farb A, Malcom GT, Liang $Y-H$, Smialek J, and Virmani $\mathrm{R}$ : Coronary risk factors and plaque morphology in men with coronary disease who died suddenly. $\mathrm{N}$ Engl J Med, 336 : 1276-1282, 1997

(19) Frost PH, Davis BR, Burlando AJ, Curb JD, Guthrie Jr GP, Isaacsohn JL, Wassertheil-Smoller $\mathrm{S}$, Wilson $\mathrm{AC}$, and Stamler J, for the Systolic Hypertension in the Elderly Research Group : Coronary heart disease risk factors in men and women aged 60 years and older. Finding from the Systolic Hypertension in the Elderly Program. Circulation, 94 : 26-34, 1996

(20) Bondjers G, Glukhova M, Hansson GK, Postnov YV, Reidy MA, and Schwartz SM : Hypertension and atherosclerosis: cause and effect, or two effects with one unknown cause? Circulation, 84(Suppl VI) : VI-2-VI-16, 1991

(21) Campbell JH and Campbell GR: Cell biology of atherosclerosis. J Hypertens, 12(Suppl 10) : S129-S132, 1994

(22) Yutani $\mathrm{C}$, Fujita $\mathrm{H}$, Imakita $\mathrm{M}$, Ishibashi-Ueda $\mathrm{H}$, Kyotani $\mathrm{S}$, Hao H, Sikino $\mathrm{T}$, and lida $\mathrm{K}$ : Coronary atherosclerosis and diabetes mellitus: A pathological study. In: Current advances in triglycerides and atherosclerosis. eds by Yamamoto $\mathrm{Y}$, Nakamura $\mathrm{H}$, and Nakai T, pp 163-171, Churchill Livingstone, Japan, 1994

(23) Heitzer T, YläHerttuala S, Luoma J, Kurz S, Müzel T, Just $\mathrm{H}$, Olschewski $M$, and Drexler $H$ : Cigarette smoking potentiates endothelial dysfunction of forearm resistance vessels in patients with hypercholesterolemia. Role of oxidized LDL. Circulation, 93 : 1346-1353, 1996

(24) Hung J, Lam JY, Lacoste L, and Letchacovski G : Cigarette smoking acutely increases platelet thrombus formation in patients with coronary artery disease taking aspirin. Circulation, 92 : 2432-2436, 1995

(25) Waters D, Lespéance J, Gladstone P, Boccuzzi SJ, Cook $T$, Hudgin R, Kilip G, and Higginson L, for the CCAIT Study Group : Effects of cigarette smoking on the angiographic evolution of coronary atherosclerosis. A Canadian Coronary Atherosclerosis Intervention Trial (CCAIT) Substudy. Circulation, 94 : 614-621, 1996

(26) Terres W, Becker P, and Rosenberg A: Changes in cardiovascular risk profile during the cessation of smoking. Am J Med, 97 : 242-249, 1994

(27) Castelli WP, Garrison RJ, Wilson PWF, Abbott RD, Kalousian S, and Kannel WB: Incidence of coronary heart disease and lipoprotein cholesterol levels. The Framigham Study. JAMA, 256 : 2835-2838, 1986

(28) Stamler J, Wentworth D, and Neaton JD, for the MRFIT Research Group: Is relationship between serum cholesterol and risk of premature death from coronary heart disease continuous and graded? JAMA, 256: 2823 2828, 1986

(29) Frost PH, Davis BR, Burlando AJ, Curb JD, Guthrie Jr GP, Isaacsohn JL, Wassertheil-Smoller $\mathrm{S}$, Wilson $\mathrm{AC}$, and Stamler J, for the Systolic Hypertension in the Elderly Research Group: Serum lipids and incidence of coro- 
nary heart disease. Finding from the Systolic Hypertension in the Elderly Program (SHEP). Circulation, 94: 2381-2388, 1996

(30) Wagner WD, St Clair RW, Clarkson TB, and Connor JR : A study of atherosclerosis regression in macaca mulatta. III. Chemical changes in arteries from animals with atherosclerosis induced for 19 months and regressed for 48 months at plasma cholesterol concentrations of 300 or $200 \mathrm{mg} / \mathrm{dl}$. Am J Pathol, 100 : 633-650, 1980

(31) Brown BG, Zhao X-Q, Sacco DE, and Albers JJ: Lipid lowering and plaque regression: new insights into prevention of plaque disruption and clinical events in coronary disease. Circulation, $87:$ 1781-1791, 1993 